12th International Symposium on Cosmology and

Particle Astrophysics (CosPA 2015)

International Journal of Modern Physics: Conference Series

Vol. 43 (2016) 1660195 (8 pages)

(C) The Author(s)

DOI: $10.1142 / \mathrm{S} 2010194516601952$

\title{
The ATLAS Detector: Status and Performance in Run-II
}

\author{
Steven Schramm \\ On behalf of the ATLAS Collaboration \\ Département de physique nucléaire et corpusculaire, Université de Genève, \\ Quai Ernest-Ansermet 24, CH-1211 Geneva, Switzerland \\ steven.schramm@unige.ch
}

Published 7 July 2016

\begin{abstract}
During the first extended shutdown of the LHC, in 2013 and 2014, the ATLAS detector has undergone several improvements. A new silicon pixel detector layer has been added inside of the existing layers, enhancing vertex identification, while the coverage of the muon detector has been significantly expanded. Many other detector systems have been upgraded to handle the higher expected pileup conditions in the coming years and to generally improve their performance. This document describes these upgrades and the resulting impact on the reconstruction and performance of standard physics objects. Preliminary results using the first $\sim 80 \mathrm{pb}^{-1}$ of 2015 data at $\sqrt{s}=13 \mathrm{TeV}$ are presented, demonstrating the capability of ATLAS to perform both searches and measurements.
\end{abstract}

Keywords: LHC; ATLAS; Run-II; Run-2; performance.

\section{Introduction}

The $\mathrm{LHC}^{1}$ has now resumed operations after the completion of the first long shutdown. The collision energy of the LHC has been increased to $\sqrt{s}=13 \mathrm{TeV}$ and the machine is preparing to deliver higher instantaneous luminosity and thus higher pileup conditions to the experiments in the near future.

The ATLAS Experiment ${ }^{2}$ has made use of the long shutdown to upgrade many detector systems, both to generally improve performance and to handle the higher expected pileup conditions. In particular, the muon detector coverage has been increased substantially and a new silicon pixel layer, named the Insertable B-Layer (IBL), has been added within the pre-existing tracking detector. The close proximity of the IBL to the collision point significantly improves the vertex reconstruction precision and tracking performance.

This is an Open Access article published by World Scientific Publishing Company. It is distributed under the terms of the Creative Commons Attribution 4.0 (CC-BY) License. Further distribution of this work is permitted, provided the original work is properly cited. 


\section{S. Schramm}

The ability of the ATLAS Collaboration to perform physics depends on:

(i) The performance of the LHC (beam energy, luminosity, bunch spacing, etc)

(ii) The functionality and coverage of the ATLAS detector

(iii) The capability of the trigger to record interesting events

(iv) The robustness of the object reconstruction and performance

(v) The techniques used in the physics searches or measurements

This document focuses on items (ii) through (iv), thus covering the improvements made by ATLAS over the shutdown and their impact on the physics objects used in all physics analyses. Physics results using the first $\sim 80 \mathrm{pb}^{-1}$ of 2015 data are presented, demonstrating the capability of ATLAS to perform both searches and measurements.

\section{Detector upgrades}

The detector systems have received a significant overhaul, from improvements to the cryogenics and other support services to replacements of pixel modules and calorimeter electronics. A more substantial change is the completed installation of the muon systems, which significantly improves the muon detector coverage.

Possibly the largest upgrade to the detector over the long shutdown was the addition of the IBL. This new layer of silicon pixels lies within the existing tracking detector, and is located only $3.3 \mathrm{~cm}$ from the beam. This is significantly closer than the previously innermost pixel layer, which is $5.0 \mathrm{~cm}$ from the beam, and was made possible thanks to the use of a new (smaller) beam pipe. ${ }^{3}$ The impact of the IBL on physics objects will be discussed in Sec. 4 .

\section{Trigger and operations}

During 2015, the LHC provided bunch spacings between collisions of both $50 \mathrm{~ns}$ and $25 \mathrm{~ns}$, or collision rates of up to $20 \mathrm{MHz}$ and $40 \mathrm{MHz}$ respectively. However, the ATLAS data storage and Tier0 processing system is only capable of writing out $\sim 3 \mathrm{~GB} / \mathrm{s}$, which corresponds to recording events at approximately $1 \mathrm{kHz}$. The ATLAS multi-level trigger system is responsible for identifying the most interesting events and providing the required four order of magnitude reduction in the rate.

The Level-1 (L1) trigger is based on hardware and is used to perform a very quick pass over each event. This first pass only considers simple physics objects, such as fixed-size sliding window cluster jets, and does not allow for topological selections involving different objects. A new hardware system added over the shutdown, known as the L1 topological (L1topo) trigger, extends this functionality to allow topological selections between these simple objects such as $\Delta \phi\left(E_{\mathrm{T}}^{\mathrm{miss}}\right.$, jet $)$. Various upgrades in the L1 trigger hardware, including the installation of a new central trigger processor, has allowed for a rate increase from $75 \mathrm{kHz}$ to $100 \mathrm{kHz}$.

The second and final stage of the ATLAS trigger is the High Level Trigger (HLT). This trigger is software-based, and combines the Level-2 and Event Filter 


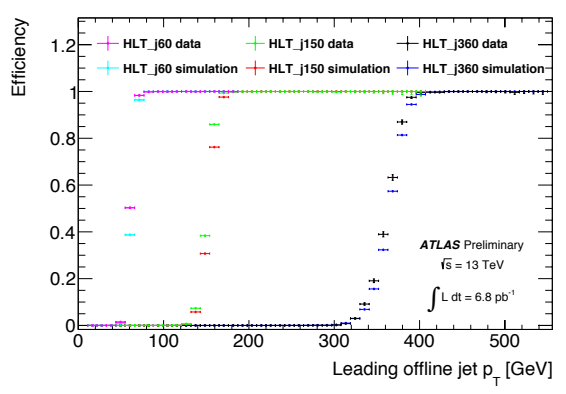

(a)

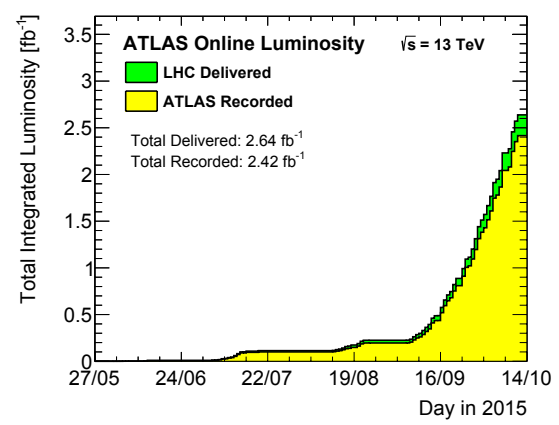

(b)

Fig. 1. (a) A series of single-jet triggers up to the lowest unprescaled trigger, which is shown to be fully efficient in both data and simulation for $p_{\mathrm{T}} \gtrsim 410 \mathrm{GeV} .{ }^{4}$ (b) The amount of cumulative luminosity delivered to and recorded by ATLAS as of October 14, 2015. This plot is originally from Ref. 5, which has since been updated to include the rest of the 2015 data taking period.

triggers used in Run-I, thus reducing the computational overhead. A considerable amount of effort went into optimizing and aligning the HLT reconstruction software with that which is done offline, resulting in sizable trigger performance gains. As an example, trigger efficiencies for a series of single-jet triggers are shown in Fig. 1(a).

More than $2.6 \mathrm{fb}^{-1}$ of data has been delivered to ATLAS, and more than $2.4 \mathrm{fb}^{-1}$ of that data has been recorded ${ }^{\mathrm{a}}$ as shown in Fig. 1(b). Of the recorded data, the first $\mathcal{O}\left(100 \mathrm{pb}^{-1}\right)$ used a bunch spacing of $50 \mathrm{~ns}$, while the remainder had a bunch spacing of $25 \mathrm{~ns}$. However, not all of the data is useable for physics. Detector downtime and other problems reduce the amount of recorded data by a few percent. ${ }^{6}$

\section{Physics object reconstruction and performance}

The reconstruction and performance of physics objects have seen large changes over the shutdown. Most of the ATLAS reconstruction software has been redesigned, and new techniques have been studied for further improvement of object performance.

\subsection{Tracking and vertexing}

The close proximity of the newly added IBL to the beam has significantly improved the tracking performance, particularly with regard to the impact parameter resolution. An improvement of roughly a factor of two can be seen in Fig. 2(a).

The first data taken in June 2015 was used to update the tracking detector alignment, improving agreement between data and MC in basic tracking distributions, as outlined in Ref. 8. After alignment, more complex distributions including the number of hits on tracks in jets are seen to be well modelled, as shown in Fig. 2(b). Such variables have many important uses, such as jet calibration and $b$-jet tagging.

${ }^{\mathrm{a}}$ At the time of writing, $4.2 \mathrm{fb}^{-1}$ of data has been delivered and $3.9 \mathrm{fb}^{-1}$ of data has been recorded. 


\section{S. Schramm}

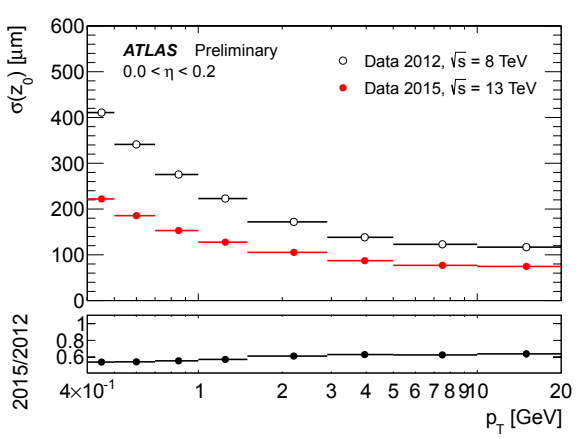

(a)

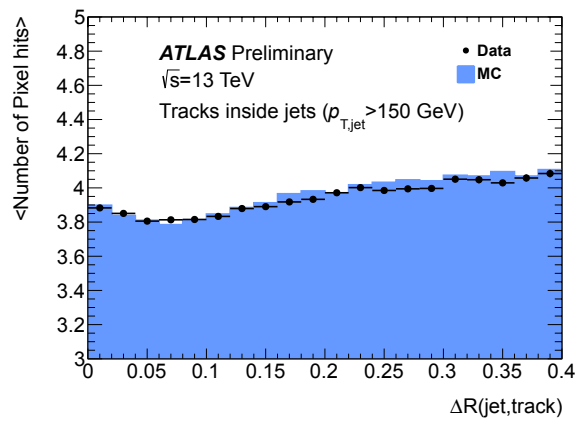

(b)

Fig. 2. (a) A comparison of the $z_{0}$ impact parameter resolution in 2012 vs 2015, demonstrating the gains made possible by the addition of the IBL. ${ }^{7}$ (b) The average number of pixel hits on tracks inside jets of $p_{\mathrm{T}}>150 \mathrm{GeV} .^{3}$

\subsection{Charged leptons}

The $J / \psi \rightarrow \mu^{+} \mu^{-}$and $Z \rightarrow \mu^{+} \mu^{-}$mass peaks have been used to probe the performance of muons in ATLAS. The reconstruction efficiency is found to be above $99 \%$ for $p_{\mathrm{T}}>7 \mathrm{GeV}$ and the scale and resolution are both seen to match the expected mass peaks within uncertainties. ${ }^{9}$ Excellent modelling of data is also observed across the muon system coverage, as shown in Fig. 3(a).

The $Z \rightarrow e^{+} e^{-}$mass peak was used to study electron performance, while $J / \psi \rightarrow$ $e^{+} e^{-}$was used as a cross-check. Electron identification is based on a likelihood involving shower shapes and tracking information, and the identification efficiency is seen to differ between data and simulation at a similar level to Run-I. This is understood to be from poor modelling of some of the shower shape variables, and is

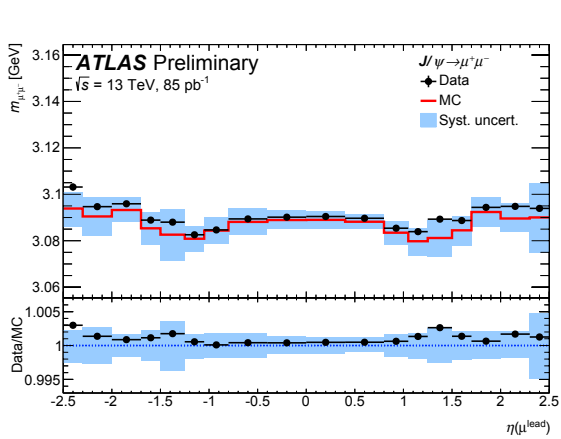

(a)

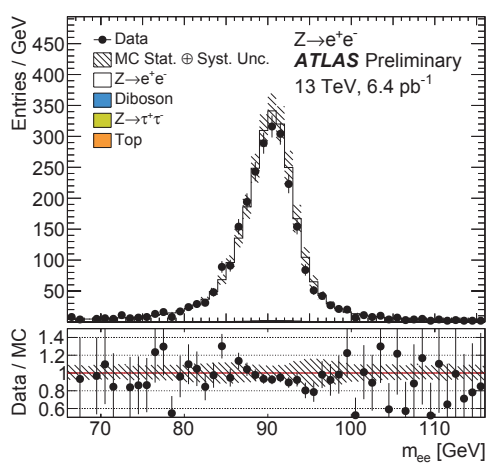

(b)

Fig. 3. (a) The mean value of the di-muon invariant mass of $J / \psi \rightarrow \mu^{+} \mu^{-}$decays, as a function of the leading muon pseudorapidity. ${ }^{9}$ (b) The di-electron invariant mass for $Z \rightarrow e^{+} e^{-}$decays. ${ }^{11}$ 
accounted for using scale factors. After applying these scale factors, the scale and resolution of electrons agree between data and simulation, as seen in Fig. 3(b).

\subsection{Jets, $b$-jets, and large- $R$ jets}

The calibration procedure for anti- $k_{t} R=0.4$ jets has been updated to provide similar performance in 2015 data, while the associated uncertainties have been extrapolated from Run-I to Run-II. This extrapolation is required until in situ techniques can be applied to 2015 data, and involves additional systematic sources for each change expected: detector read-out, reconstruction algorithms, bunch spacing, and more. ${ }^{12}$ The uncertainty for central jets is below the level of $2 \%$ from approximately $100 \mathrm{GeV}$ to $1.75 \mathrm{TeV}$ as shown in Fig. 4(a).

The $b$-jet tagging performance in 2015 has improved substantially from 2012 thanks to both the IBL and improved tagging algorithms. A comparison was conducted using $t \bar{t}$ simulated samples for jets with $p_{\mathrm{T}}>25 \mathrm{GeV}$ and $|\eta|<2.5$, and it was found that the 2015 tagger rejects four times (1.7 times) more light quark and gluon jets (charm jets) than in 2012 at the $70 \%$ signal efficiency working point. ${ }^{13}$ Fig. 4(b) demonstrates that the $b$-jet tagging algorithm in 2015 shows good agreement between data and simulation in $t \bar{t}$ events.

Taggers for anti- $k_{t} R=1.0$ jets trimmed with $f_{\text {cut }}=0.5$ and $R_{\text {sub }}=0.2$, containing hadronic decays of boosted $W / Z$ bosons and Higgs bosons, have also been studied in 2015. The $\mathrm{W} / \mathrm{Z}$ tagger is seen to have a QCD rejection factor of approximately 50 (200) for a signal efficiency of $50 \%$ (25\%). ${ }^{15}$ The $H \rightarrow b b$ tagger at an approximately $50 \%$ working point suppresses many other backgrounds at

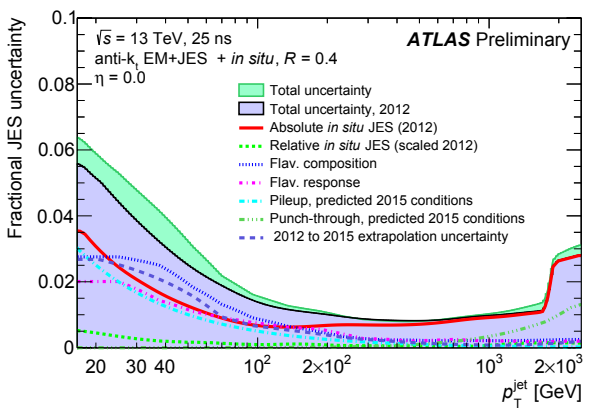

(a)

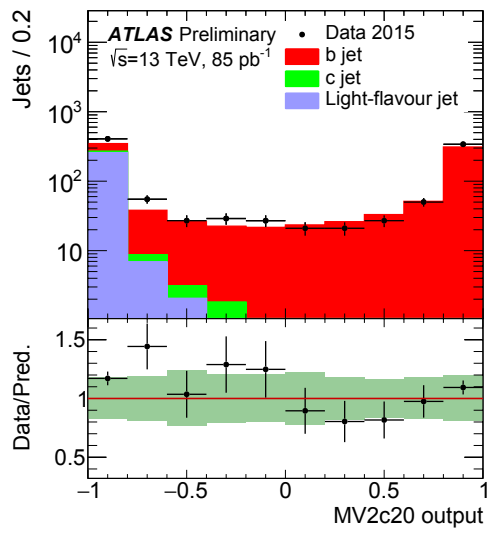

(b)

Fig. 4. (a) The jet energy scale uncertainty for central anti- $k_{t} R=0.4$ jets in the nominal $25 \mathrm{~ns}$ bunch spacing configuration. ${ }^{12}$ (b) The output distribution of the MV2c20 b-jet tagging algorithm in $t \bar{t}$ enriched data and simulated events, demonstrating good agreement. ${ }^{14}$ 


\section{S. Schramm}

many orders of magnitude, from $X \rightarrow b b$ rejection of a factor of four to light quark rejection of a factor of more than $10^{5} .{ }^{16}$

\subsection{Missing transverse momentum}

The calculation of $E_{\mathrm{T}}^{\text {miss }}$ in ATLAS proceeds in several steps. High- $p_{\mathrm{T}}$ (hard) objects are first identified and calibrated at their respective scales. The various hard objects are then combined one type at a time, starting with electrons. Overlap removal is performed as each new type of object is added to the mix in order to avoid doublecounting any calorimeter energy deposits. After all of the hard objects have been combined, the remaining low- $p_{\mathrm{T}}$ (soft) energy is combined to form the soft term.

In 2012, the soft term was built primarily from the calorimeter, and thus is known as the Calorimeter Soft Term (CST). In 2015, studies have been done to use tracking information in the soft term to form the Track Soft Term (TST). This requires matching of tracks to the hard objects to avoid double-counting energy already present in the hard terms, but has been seen to improve the resolution stability with respect to pileup and to reduce the bias in the $E_{\mathrm{T}}^{\mathrm{miss}}$ scale, as seen in Fig. 5. Studies in 2015 data using $Z \rightarrow \mu^{+} \mu^{-}$and $W \rightarrow e \nu$ events have shown that the TST definition of the $E_{\mathrm{T}}^{\text {miss }}$ is well modelled. ${ }^{18}$

\section{Physics results}

The first $85 \mathrm{pb}^{-1}$ of 2015 data already contains $\mathcal{O}\left(10^{6}\right) W^{ \pm}$and $\mathcal{O}\left(10^{5}\right) Z$ candidate events, rendering the statistical uncertainties negligible for the $W^{ \pm}$and $Z$ fiducial cross-section measurements. ${ }^{19}$ The luminosity uncertainty is currently the dominant systematic source, as seen in Fig. 6(a). If the luminosity uncertainty is ignored, the theory and other experimental uncertainties are already on the same scale. Due to

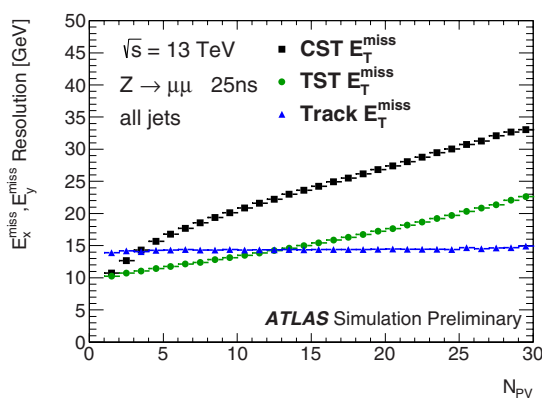

(a)

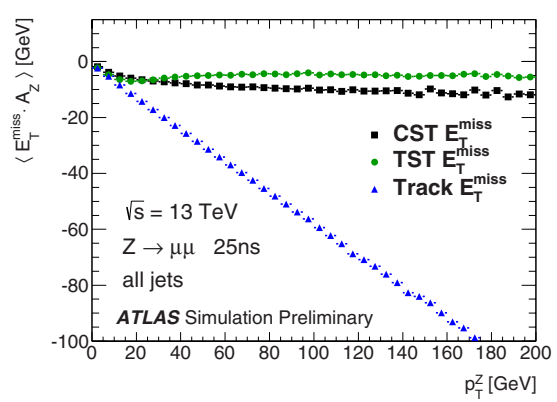

(b)

Fig. 5. (a) The $E_{\mathrm{x}, \mathrm{y}}^{\mathrm{miss}}$ resolution as a function of the number of reconstructed primary vertices, demonstrating the reduced pileup dependence of the new TST variant with respect to the CST. ${ }^{17}$ (b) The projection of the reconstructed $E_{\mathrm{T}}^{\text {miss }}$ along the $\mathrm{Z}$ boson axis in $Z\left(\rightarrow \mu^{+} \mu^{-}\right)+$jet(s) events, which have no real $E_{\mathrm{T}}^{\mathrm{miss}}$ source. A bias in the scale is observed in the jet system direction, and the new TST variant is seen to reduce the magnitude of the bias with respect to the CST. ${ }^{17}$ 


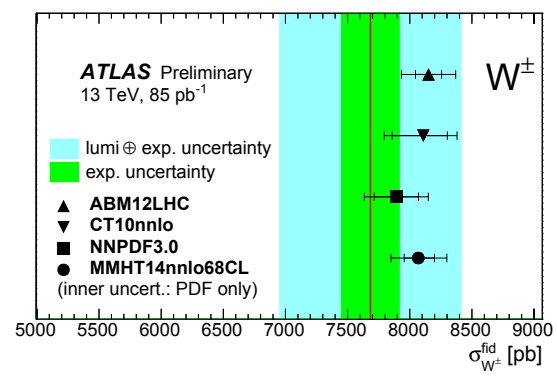

(a)

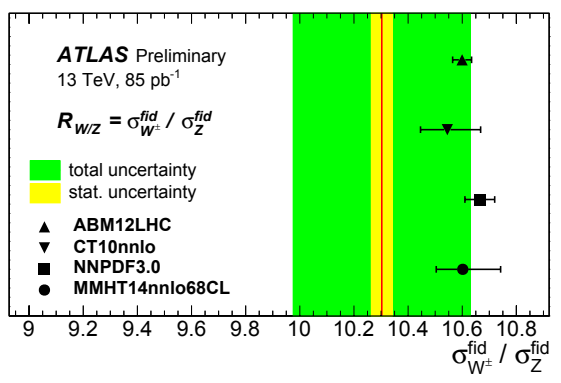

(b)

Fig. 6. The (a) $W^{ \pm}$fiducial cross-section and (b) the ratio of the $W^{ \pm}$to $Z$ fiducial cross sections with the first $85 \mathrm{pb}^{-1}$ of $\sqrt{s}=13 \mathrm{TeV}$ data. ${ }^{19}$ Neglecting the luminosity uncertainty, the theory and experimental uncertainties are already comparable.

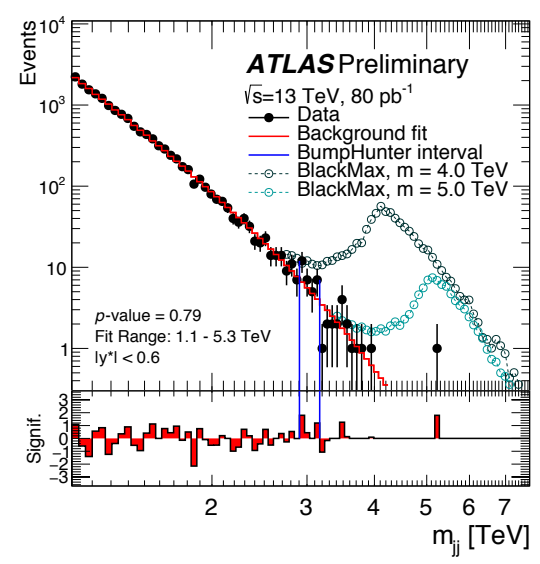

Fig. 7. The invariant mass spectrum from the dijet resonance search as conducted with the first $80 \mathrm{pb}^{-1}$ of $\sqrt{s}=13 \mathrm{TeV}$ data. ${ }^{20}$ The search is already probing new regions of parameter space. This result has since been superseded with a result from the full 2015 dataset. ${ }^{21}$

the cancellation of the luminosity uncertainty when taking the ratio of the $W^{ \pm} / Z$ fiducial cross sections, the measurement is already starting to probe the theoretical predictions, as seen in Fig. 6(b).

The dijet resonance search is very sensitive to the center of mass energy, and thus $80 \mathrm{pb}^{-1}$ of $\sqrt{s}=13 \mathrm{TeV}$ is sufficient to study new regions of parameter space compared to the full $\sqrt{s}=8 \mathrm{TeV}$. This is particularly true for models of black hole production, as shown in Fig. 7 , while other models require $\mathcal{O}\left(1 \mathrm{fb}^{-1}\right)$ of data before the 2012 sensitivity can be surpassed. No excess was observed in the first $80 \mathrm{pb}^{-1}$. 


\section{S. Schramm}

\section{Conclusions}

The ATLAS Collaboration has taken advantage of the long shutdown in order to upgrade the detector services and electronics, finish the installation of the muon system, and install a new layer of silicon pixels. The trigger systems have been restructured and a new hardware has enabled higher processing rates. The physics object reconstruction software has been substantially redesigned, and many new algorithms are in use. The combination of the upgraded detector and reconstruction algorithms have improved the physics object performance in many areas, which has been confirmed in the early 2015 dataset.

Physics analyses stand to benefit from these many improvements. The first measurements are already pushing theoretical predictions and the first searches have surpassed the 2012 dataset's sensitivity to some models of physics beyond the standard model. The ATLAS Experiment has exited the first long shutdown of the LHC in excellent shape, and is ready to push the physics reach even further as more data becomes available.

\section{References}

1. L. Evans and P. Bryant (eds.), JINST 3, S08001 (2008).

2. ATLAS Collaboration, JINST 3, S08003 (2008).

3. ATLAS Collaboration, ATL-PHYS-PUB-2015-018 (2015).

4. ATLAS Collaboration, Jet Trigger Performance Plots, (July 2015). https://twiki.cern.ch/twiki/bin/view/AtlasPublic/JetTriggerPublicResults\#Jet_ Trigger_Performance_Plot_AN1

5. ATLAS Collaboration, Luminosity summary plots for 2015 data, (October 2015). https://twiki.cern.ch/twiki/bin/view/AtlasPublic/LuminosityPublicResultsRun2\# Luminosity_summary_plots_for_201

6. ATLAS Collaboration, ATLAS Run-2 Detector Status, (April 2015). https://twiki.cern.ch/twiki/bin/view/AtlasPublic/ApprovedPlotsATLASDetector \#ATLAS_Run_2_Detector_Status_from

7. ATLAS Collaboration, IDTR-2015-007 (2015).

8. ATLAS Collaboration, ATL-PHYS-PUB-2015-031 (2015).

9. ATLAS Collaboration, ATL-PHYS-PUB-2015-037 (2015).

10. ATLAS Collaboration, ATL-PHYS-PUB-2015-041 (2015).

11. ATLAS Collaboration, ATL-PHYS-PUB-2015-021 (2015).

12. ATLAS Collaboration, ATL-PHYS-PUB-2015-015 (2015).

13. ATLAS Collaboration, ATL-PHYS-PUB-2015-022 (2015).

14. ATLAS Collaboration, ATL-PHYS-PUB-2015-039 (2015).

15. ATLAS Collaboration, ATL-PHYS-PUB-2015-033 (2015).

16. ATLAS Collaboration, ATL-PHYS-PUB-2015-035 (2015).

17. ATLAS Collaboration, ATL-PHYS-PUB-2015-023 (2015).

18. ATLAS Collaboration, ATL-PHYS-PUB-2015-027 (2015).

19. ATLAS Collaboration, ATLAS-CONF-2015-039 (2015).

20. ATLAS Collaboration, ATLAS-CONF-2015-042 (2015).

21. ATLAS Collaboration, PRL B 754, 302 (2016). 